\title{
A Queueing Model for a Wireless GSM/GPRS Cell with Multiple Service Classes
}

\author{
D.D. Kouvatsos, K. Al-Begain, and I. Awan \\ Department of Computing, School of Informatics, University of Bradford \\ BD7 1DP, Bradford, West Yorkshire, England, UK \\ \{d.d.kouvatsos, k.begain, i.awan\}@bradford.ac.uk
}

\begin{abstract}
A novel analytic framework is devised for the performance modelling and evaluation of a wireless cell using Global System for Mobile telecommunication (GSM) with General Packet Radio Service (GPRS) supporting both voice and multiple class data services, respectively, under a complete partitioning scheme (CPS). In this context, a queueing model is proposed consisting of two independent queueing systems, namely an $\mathrm{M} / \mathrm{M} / \mathrm{c} / \mathrm{c}$ loss system with Poissonian GSM traffic and a $\left\{\mathrm{GE} / \mathrm{GE} / 1 / \mathrm{N}_{1} / \mathrm{FCFS} \rightarrow \mathrm{GE} / \mathrm{GE} / 1 / \mathrm{N}_{2} / \mathrm{PS}\right\}$ system of access and transfer finite capacity queues in tandem having an external Compound Poisson GPRS traffic with geometrically distributed batches and generalised exponential (GE) service times under first-come-first-served (FCFS) and processor sharing (PS) scheduling rules, respectively. Although the analysis of the former loss system is straightforward, the solution of the GE-type queues in tandem is rather complex. This investigation focuses on the analysis of the tandem GE-type queueing system, which is valid for both uplink and downlink connections and provides multiple class data services with different arrival rates, interarrival-time squared co-efficient of variations (SCVs), file (burst) sizes and PS discrimination service levels. The principle of maximum entropy (ME) is used to characterise a product form approximation, subject to appropriate GE-type queueing theoretic constraints per class, and thus, implying a decomposition of the tandem system into $\mathrm{GE} / \mathrm{GE} / 1 / \mathrm{N}_{1} / \mathrm{FCFS}$ and $\mathrm{GE} / \mathrm{GE} / 1 / \mathrm{N}_{2} / \mathrm{PS}$ building block queues, each of which can be analysed in isolation. Subsequently, closed form expressions for state and blocking probabilities are obtained. Typical numerical examples are included to validate the ME solution against simulation and study the effect of external GPRS bursty traffic upon the performance of the cell.
\end{abstract}

Keywords: Cellular mobile system, Global System for Mobile Telecommunication (GSM), General Packet Radio Service (GPRS), wireless GSM/GPRS cell, complete partitioning scheme (CPS), performance evaluation, maximum entropy (ME) principle, generalised exponential (GE) distribution, first-come-first-served (FCFS) rule, processor sharing (PS) rule. 


\section{Introduction}

Queueing theoretic models are widely recognised as powerful and realistic tools for the performance evaluation and prediction of complex mobile systems. However, there are inherent difficulties and open issues to be resolved before a global network infrastructure for broadband mobile systems can be established. Some of these problems may be attributed to the complexity of mobile traffic characterisation and the assessment of its performance impact based on the much needed derivation of closed form metrics. Most of the published performance studies in the field are based on simulation modelling and numerical solution of Markov models covering different traffic scenarios, mostly at call level, with single or multiple service classes. Earlier proposed models are based on resource network management parameters of the Global System for Mobile Telecommunications (GSM) technology, where the capacity of radio interference in the wireless cell is divided into discrete channels and operates in circuit-switched mode (e.g., [1]. More recently, extensions of these models have been made to capture the packetswitched behaviour introduced by the General Packet Radio Service (GPRS) which has been added to GSM to allow data communication with higher bit rates than those provided by a single GSM channel (e.g., [2,3]). More recently, Foh et al [4] proposed a single server infinite capacity queue for modelling GPRS in a Markovian environment and applied matrix geometric methods for the evaluation of performance metrics.

Simulation is an efficient tool for studying detailed system behaviour but it becomes costly, particularly as the system size increases. Markov models on the other hand provide more flexibility and produce numerical results for many interesting performance measures. Nevertheless, the numerical solution of Markov models may suffer from several drawbacks, such as

- state space explosion limiting the analysis to only small mobile systems, generally consisting of one cell,

- restrictive assumptions of independent Poisson arrival processes for all types of homogeneous and uniformly distributed traffic with exponentially distributed call durations (which, if multiplexed, can be bursty and correlated).

Thus, there is still a great need to consider alternative analytic methodologies for the analysis of queueing models, based on a balanced trade-off between simplified assumptions to reduce complexity and actual real life system behaviour, leading to both credible and cost-effective approximations for the performance prediction and optimisation of mobile systems.

This investigation proposes a novel analytic framework for the performance modelling and evaluation of a wireless GSM/GPRS cell with both voice and multiple data services under a complete partitioning scheme (CPS). The work focuses on the analysis of a tandem generalised exponential (GE)-type queueing model involving a first-come-firs-served (FCFS) access queue and a discriminatory processor sharing (PS) transfer queue (air interface) with distinct multiple data service classes and external Compound Poisson GPRS (multiplexed) traffic class streams with geometrically distributed batches. The model is analysed 
via the principle of maximum entropy (ME) (c.f., $[5,6]$ ) which is used to characterise a product form approximation, subject to GE-type queueing theoretic constraints, and thus, allowing system decomposition and the separate analysis of each of the two GE-type queues in tandem. Subsequently, closed form expressions for state and blocking probabilities per class are obtained.

The paper is organised as follows. Section 2 describes call handling schemes for wireless GSM/GPRS cells. The GE-type tandem queueing model is discussed in Section 3 together with the characterisation of an ME product form approximation. Section 4 presents the ME analysis of the GE/GE/1/N building block queue with either FCFS or discriminatory PS scheduling rules. Numerical examples to validate the ME solution against simulation and study the effect of external GPRS bursty traffic upon the performance of the cell are included in Section 5. Concluding remarks follow in Section 6.

\section{GSM/GPRS Call Handling Schemes}

Resources for GPRS traffic can be reserved statically or dynamically, whereas a combination of both is possible. Different partitioning schemes can be defined where partitions are created for GSM and GPRS traffic but not for individual data services. For GPRS traffic, a complete partition is used for different data services. However, some data calls may be allocated higher priority and therefore they can be given higher share of the available bandwidth. Whenever voice and data share bandwidth, voice service is always given the highest priority. Two main partitioning schemes, namely complete partitioning and partial sharing, are described below:

- Complete partitioning scheme (CPS) divides the total cell capacity to serve simultaneously GSM and GPRS traffics. As a consequence, the GSM and GPRS systems can be analysed separately.

- Partial sharing scheme (PSS) allocates $C_{d a t a}$ channels for data traffic and the remaining $C_{\text {shared }}=C_{\text {total }}-C_{\text {data }}$ channels are shared by voice and data calls with preemptive priority for voice calls.

CPS has the advantage of requiring simpler management policy and implementation. Moreover, a definite capacity for GPRS under an efficient Connection Admission Control (CAC) algorithm can make feasible some QoS guarantees, although it will not clearly give the best utilisation for radio resources. Note that the CPS is the limiting case of the PSS under high loads. The GSM partition can be clearly modelled as a loss system. An admitted GSM voice call needs the assignment of a single traffic channel for its entire duration. On the absence of an available channel, a voice call is lost. Moreover, the GPRS partition can be represented by a finite capacity queueing model involving two single server queues in tandem, namely a FCFS access queue and a discriminatory PS transfer queue, where all active data connections share the total capacity of the data partition and may belong to various classes. These classes may have different 
characteristics such as maximum or minimum data rates, delay sensitivity, service discrimination, arrival rates, interrival-time variability and transferable file (data) length.

A transfer queue holds a finite number of data connections which are served according to a discriminatory PS rule, where the available service capacity is shared evenly amongst all data calls belonging to the same class. However, in the presence of multiple data classes with different priority levels, the service capacity is shared according to discrimination rates favouring higher priority classes. An admitted data call is initially held in a finite capacity FCFS access queue. If the access queue is full, the incoming call is lost. The access queue models the Packet Control Unit (PCU)/Sevicing GPRS Support Node (SGSN) buffers of a GPRS network with down-link traffic or the logical queue of data call request for transmission in the up-link stream. A call at the head of the access queue will be blocked if the transfer queue is full. Upon the termination of an active data call at the transfer queue, the blocked data call at the access queue is polled into the transfer queue (within a very short time required for signaling) and immediately shares in a PS fashion the available capacity.

\section{The GE-Type Tandem Queueing Model}

This section introduces a queueing network model for the performance analysis of a wireless GSM/GPRS cell with both voice and multiple data services under CPS. The model describes the GSM and GPRS partitions which can be studied separately (c.f., Section 2). Assuming a Poissonian arrival process, the GSM partition can be modelled by the classical Birth-and-Death $\mathrm{M} / \mathrm{M} / \mathrm{c} / \mathrm{c}$ loss system with exponential call durations (which can be analysed via Erlang's loss formula).

The GPRS partition on the other hand can be modelled as a tandem GE-type $\mathrm{GE} / \mathrm{GE} / 1 / \mathrm{N}_{1} / \mathrm{FCFS} \rightarrow \mathrm{GE} / \mathrm{M} / 1 / \mathrm{N}_{2} / \mathrm{PS}$ finite capacity queueing system, where both external and internal traffics are approximated by GE-type interarrivaltime distributions, or equivalently, Compound Poisson arrival processes, respectively, with geometrically distributed batches (c.f., Fig.1). Under PS rule, $\mathrm{N}_{2}$ represents the maximum number of connections sharing simultaneously the available service capacity. Note that a batch arrival process is a most suitable model of bursty multiplexed connections (belonging to various classes with different minimum capacity demands) being accepted into the mobile system if there is enough service capacity at the moment of their arrival. Although the stochastic analysis of this GE-type tandem system is rather complex, the principle of maximum entropy (ME) can be used, as in earlier works [5,6], to characterise a product form approximation, subject to appropriate GE-type marginal queueing theoretic constraints.

More specifically, the form of the ME joint state probability $P(\mathbf{k}), \mathbf{k}=$ $\left(\mathbf{k}_{1}, \mathbf{k}_{2}\right)$ of the tandem system, where $\mathbf{k}_{j}$ is a state vector $\left(k_{j 1}, \ldots, k_{j R}\right)$ and $k_{j i}$ is the number of calls of class $i$ in queue $j$ for $i=1, \ldots, R$ and $j=1$ (access queue), 2 (transfer queue), subject to normalisation and the existence of the marginal constraints of server utilisation, mean queue length and full buffer 


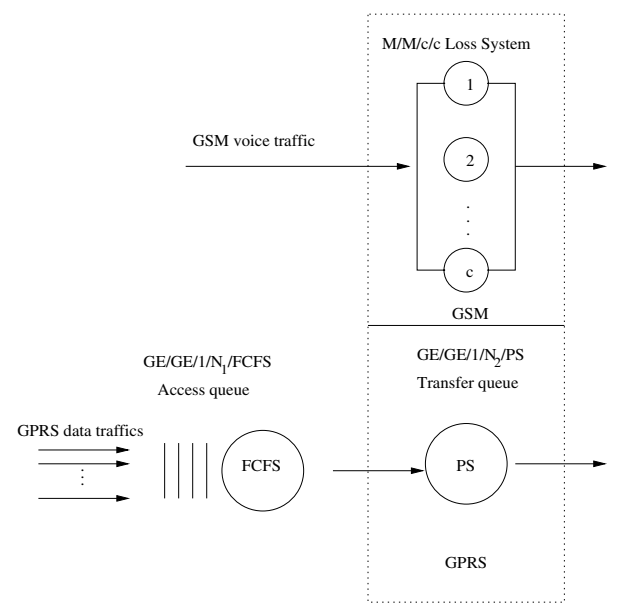

Fig. 1. The Wireless GSM/GPRS with CPS

state probability per class, can be clearly established by applying the method of Lagrange's undetermined multipliers and is given by

$$
P(\mathbf{k})=P_{1}\left(\mathbf{k}_{1}\right) P_{2}\left(\mathbf{k}_{2}\right)
$$

where $P_{1}\left(\mathbf{k}_{1}\right)$ and $P_{2}\left(\mathbf{k}_{2}\right)$ are the marginal joint state (or, queue length) probabilities of the GE/GE/1/N $/$ FCFS access queue and GE/GE/1/ $\mathrm{N}_{2} / \mathrm{PS}$ transfer queue, respectively. This product form approximation allows the decomposition of the tandem system into the two aforementioned queues, each of which can be solved in isolation by carrying out ME analysis at the queue level in conjunction with flow formulae relating, approximately, to a GE-type interdeparture-time mean and SCV (c.f., Kouvatsos et al [5]), namely

$$
\lambda_{d 1 i}=\lambda_{1 i}, C_{d 1 i}^{2}=2\left\langle n_{1 i}\right\rangle p_{1 i}(0)-C_{a 1 i}^{2}\left(1+p_{1 i}(0)\right),
$$

where $\left\{\left\langle n_{1 i}\right\rangle, i=1,2, \ldots, R\right\}$ are the marginal mean queue lengths of the access queue $\mathrm{GE} / \mathrm{GE} / 1 / \mathrm{N}_{1} / \mathrm{FCFS}$ and $\left\{p_{1 i}(0), i=1,2, \ldots, R\right\}$ are the marginal probabilities that there are no data calls of class $i, i=1,2, \ldots, R$, in the access queue.

Note that the proposed $\left\{\mathrm{GE} / \mathrm{GE} / 1 / \mathrm{N}_{1} / \mathrm{FCFS} \rightarrow \mathrm{GE} / \mathrm{GE} / 1 / \mathrm{N}_{2} / \mathrm{PS}\right\}$ tandem queueing model with multiple service classes and blocking differs from and in some respect extends overall the $\mathrm{MMPP} / \mathrm{M} / \mathrm{c}$ queueing model suggested by Foh et al [4]. Although the later incorporates a PSS, Markov Modulated Poisson Process (MMPP) and multiple channels, nevertheless it is only applicable to a single service class, assumes exponential transmission times and, being an infinite capacity queueing model, does not capture the adverse effect of blocking on system performance. Moreover, the GE-type queueing models can be solved via closed form expressions as opposed to computationally expensive matrix geometric methods. 


\section{The ME Analysis of a GE/GE/1/N/\{FCFS or PS $\}$ Queue}

This section applies entropy maximisation to analyse a generic GE/GE/1/N queueing model, as a building block queue, with $\mathrm{R}(>0)$ classes of jobs, censored arrival processes, finite buffer capacity, $\mathrm{N}$, complete buffer management scheme and either FCFS or PS service rules. Note that the GE distribution is of the form (c.f., $[5,6])$

$$
F(t)=P(X \leq t)=1-\tau e^{-\tau v t}, t \geq 0
$$

where $\tau=2 /\left(C^{2}+1\right), X$ is the inter-event time random variable and $\left\{1 / v, C^{2}\right\}$ are the mean and squared coefficient of variation (SCV) of the inter-event time distribution, respectively. Moreover, the underlying counting process of the GE distribution is a compound Poisson process with geometrically distributed batch sizes and mean batch size $1 / \tau=\left(C^{2}+1\right) / 2$.

\section{Notation}

Without the loss of generality and for the sake of simplifying the notation, the subscript $j, j=1,2$, referring to access and transfer queues, respectively, is dropped from the notation of this section. Let at any given time

$\mathbf{S}=\left(c_{1}, c_{2}, \ldots, c_{n}\right), n \leq N$ be a joint system state, where $c_{1}$ is the class of the job in service and $c_{\ell} \in\{1,2, \ldots, R\}, \ell=2,3, \ldots, n$ is the class of $\ell^{t h}$ job in the queue, $\mathbf{Q}$ be the set of all feasible states of $\mathbf{S}$ and $P(\mathbf{S})$ be the stationary state probability.

For each class $i, i=1,2, \ldots, R$ let

$\lambda_{i}$ be the arrival rate, $\mu_{i}$ be the service rate and $\pi_{i}$ be the blocking probability that an arrival of class $i$ finds the queue full.

For each state $\mathbf{S}, \mathbf{S} \in \mathbf{Q}$, and class $i, i=1,2, \ldots, R$, the following auxiliary functions are defined:

$$
\begin{aligned}
& n_{i}(\mathbf{S})=\text { the number of class } i \text { customers present in state } \mathbf{S}, \\
& s_{i}(\mathbf{S})=1, \text { if the job in service is of class } i \text { or } 0, \text { otherwise, } \\
& f_{i}(\mathbf{S})=1 \text {, if } \sum_{i=1}^{R} n_{i}(\mathbf{S})=N \text {, and } s_{i}(\mathbf{S})=1 \text { or } 0 \text {, otherwise. }
\end{aligned}
$$

The form of the state probability distribution, $P(\mathbf{S}), \mathbf{S} \in \mathbf{Q}$, can be characterised by maximising the entropy functional $H(\mathbf{P})=-\sum_{\mathbf{S}} P(\mathbf{S}) \log P(\mathbf{S})$, subject to normalisation and server utilisation, mean queue length and full buffer state probability constraints per class satisfying the flow balance equations, namely

$$
\lambda_{i}\left(1-\pi_{i}\right)=\mu_{i} U_{i}, i=1, \ldots, R .
$$

By employing Lagrange's method of undetermined multipliers the following solution is obtained

$$
P(\mathbf{S})=\frac{1}{Z} \prod_{i=1}^{R} g_{i}^{s_{i}(\mathbf{S})} x_{i}^{n_{i}(\mathbf{S})} y_{i}^{f_{i}(\mathbf{S})}, \forall \mathbf{S} \in \mathbf{Q},
$$


where $Z$ is the normalising constant and $\left\{g_{i}, x_{i}, y_{i}, i=1,2, \ldots, R\right\}$ are the Lagrangian coefficients corresponding to the server utilisation, mean queue length and full buffer state probability constraints per class, respectively. Defining the sets

$$
\begin{aligned}
S_{0} & =\left\{\mathbf{S} / \mathbf{S} \in \mathbf{Q}: s_{i}(\mathbf{S})=0, i=1,2, \ldots, R\right\}, \\
Q_{i} & =\left\{\mathbf{S} / \mathbf{S} \in \mathbf{Q}: s_{i}(\mathbf{S})=1, i=1,2, \ldots, R\right\}, \\
Q_{i ; \mathbf{k}} & =\left\{\mathbf{S} \in Q_{i}: n_{i}(\mathbf{S})=k_{i} \& k_{i} \geq 1, i=1, \ldots, R\right\},
\end{aligned}
$$

and aggregating $P(\mathbf{S})$ over all feasible states $\mathbf{S} \in \mathbf{Q}$, the joint 'aggregate' state ME solution is given by

$$
\begin{aligned}
P\left(S_{0}\right) & =\frac{1}{Z} \\
P(\mathbf{k}) & =\sum_{i=1}^{R} \operatorname{Prob}\left(Q_{i ; \mathbf{k}}\right) \\
& =\frac{1}{Z} \frac{\left(\sum_{j=1}^{R} k_{j}-1\right) !}{\prod_{j=1}^{R} k_{j} !}\left(\prod_{j=1}^{R} x_{j}^{k_{j}}\right)\left(\sum_{i=1}^{R} k_{i} g_{i} y_{i}^{\delta(\mathbf{k})}\right),
\end{aligned}
$$

where $\delta(\mathbf{k})=1$, if $\sum_{i} k_{i}=N$, or 0 , otherwise, $\mathbf{k}=\left(k_{1}, k_{2}, \ldots, k_{R}\right)$ and $k_{i}$ be the number of jobs of class $i$ present in the queue, $i=1,2, \ldots, R$.

By using equations (6) and (7), closed form expressions for the aggregate state probabilities $\left\{P_{N}(n), n=0,1, \ldots, N\right\}$ and marginal state probabilities $\left\{P_{i}(k), k=0,1, \ldots, N_{i}, i=1,2, \ldots, R\right\}$ can be obtained (c.f., [7]). Moreover, the Lagrangian coefficients $x_{i}$ and $g_{i}$ can be approximated analytically by making asymptotic connections to the corresponding GE-type infinite capacity queue. Assuming $x_{i}$ and $g_{i}$ are invariant to the buffer capacity size $N$, it can be established that

$$
x_{i}=\frac{\left\langle n_{i}\right\rangle-\rho_{i}}{\langle n\rangle}, g_{i}=\frac{(1-X) \rho_{i}}{(1-\rho) x_{i}},
$$

where $X=\sum_{i=1}^{R} x_{i},\langle n\rangle=\sum_{i=1}^{R}\left\langle n_{i}\right\rangle$ and $\left\langle n_{i}\right\rangle$ is the asymptotic marginal mean queue length of a multi-class GE/GE/1 queue. Note that closed form expressions for $\left\{\left\langle n_{i}\right\rangle, i=1,2, \ldots, R\right\}$ have been determined in Kouvatsos et al [5]) and are given by

$$
\begin{gathered}
\left\langle n_{i}\right\rangle=\frac{\rho_{i}}{2}\left(C_{a i}^{2}+1\right)+\frac{1}{2(1-\rho)} \sum_{j=1}^{R} \frac{\lambda_{i}}{\lambda_{j}} \rho_{j}^{2}\left(C_{a j}^{2}+C_{s j}^{2}\right),\{\text { for FCFS rule }\} \\
\left\langle n_{i}\right\rangle=\rho_{i}\left\{C_{a i}^{2}+\frac{1}{1-\rho} \sum_{j=1}^{R} \frac{h_{j}}{h_{i}} \rho_{j} C_{a j}^{2}\right\},\{\text { for PS rule }\}
\end{gathered}
$$


where $\rho_{i}=\lambda_{i} / \mu_{i}, \rho=\sum_{i=1}^{R} \rho_{i}$ and $h_{i}, i=1,2, \ldots, R$, is a set of discriminatory weights that impose service discrimination to different priority classes.

Moreover, the blocking probabilities $\left\{\pi_{i}, i=1,2, \ldots, R\right\}$ of a $\mathrm{GE} / \mathrm{GE} / 1 / \mathrm{N}$ queue can be approximated by focusing on a tagged data call within an arriving bulk and is determined by

$$
\pi_{i}=\sum_{k=0}^{N} \delta_{i}(k)\left(1-\sigma_{i}\right)^{N-k} P_{N}(k),
$$

where $\delta_{i}(k)=\frac{r_{i}}{r_{i}\left(1-\sigma_{i}\right)+\sigma_{i}}$ for $k=0$ or 1 , otherwise, $\sigma_{i}=2 /\left(1+C_{a i}^{2}\right), r_{i}=$ $2 /\left(1+C_{s i}^{2}\right)$, and $\left.\left.\left\{C_{a i}^{2}\right), C_{s i}^{2}\right)\right\}$ are the squared coefficients of variation for the interarrival and service times per class $i$, respectively, $i=1,2, \ldots, R$.

By substituting closed form expressions for the aggregate $\left\{P_{N}(n), n=\right.$ $0 \ldots N\}$ and blocking $\left\{\pi_{i}, i=1,2, \ldots, R\right\}$ probabilities into the flow balance condition (4) and after some manipulation, the following recursive relationships for the Lagrangian coefficients $\left\{y_{i}, i=1,2, \ldots, R\right\}$, can be obtained:

$$
\begin{aligned}
y_{i}^{(n)} & =\left(\frac{1-\sigma_{i}}{X}\right) y_{i}^{(n-1)}-\Theta_{1 i}\left(\frac{1-\sigma_{i}-X}{X}\right), \text { for } n \geq 2, \\
y_{i}^{(1)} & =\Theta_{1 i}-\Theta_{2 i},
\end{aligned}
$$

where $\Theta_{1 i}=\frac{1-\rho}{1-X}+\frac{\rho\left(1-\sigma_{i}\right)}{1-\sigma_{i}-X}$, and $\Theta_{2 i}=\left(1-\sigma_{i}\right)\left(\frac{1-\rho}{1-X} \delta_{i}(0)+\frac{\rho}{1-\sigma_{i}-X}\right)$.

\section{$5 \quad$ Numerical Results}

This section presents some typical numerical experiments in order to illustrate the credibility of the proposed ME solution as a simple but cost-effective performance evaluation tool for assessing the effect of external GPRS traffic at the $\mathrm{GE} / \mathrm{GE} / 1 / \mathrm{N}_{1} / \mathrm{FCFS}$ access queue and its propagation into the $\mathrm{GE} / \mathrm{GE} / 1 / \mathrm{N}_{2} / \mathrm{PS}$ transfer queue in terms of the magnitude of the call rates and associated interarrival time squared coefficients (SCVs) of variation.

The numerical study focuses on two data service classes with different average sizes of 62.5 KBytes (class-1) and 12.5 KBytes (class-2) in conjunction with a range of corresponding SCVs, respectively. Note that these two classes may represent two typical Internet applications with different parameters, such as web browsing and email, respectively. It is assumed that the GPRS partition consists of one frequency providing total capacity of $171.2 \mathrm{Kbps}$. Among the different performance parameters that can be determined, three important ones are chosen, namely mean response time, mean queue length and blocking probability. The relative accuracy of the $\mathrm{ME}$ algorithm has been verified against simulation (QNAP-2 [8]) focusing on the performance measure of channel utilisation (c.f., Figs. 231). It can be observed that the ME results are very comparable to those obtained via simulation. 
Focusing on the GE/GE/1/N/PS queue under discriminatory PS rule favouring class 1 (service discrimination weight 1:5), it can be seen that the interarrivaltime SCV has an inimical effect, as expected, on the mean response time per class and the aggregate blocking probability (c.f., Figs. 45). Moreover, relative comparisons to assess the effects at varying degrees of interarrival time SCVs and buffer size, $\mathrm{N}$, at the GE/GE/1/N/FCFS queue upon ME generated mean queue lengths are presented in Fig. 6 and 7 respectively. It can be seen that the analytically established mean queue lengths deteriorate rapidly with increasing external interarrival-time SCVs (or, equivalently, average batch sizes) beyond a specific critical value of the buffer size which corresponds to the same mean queue length for two different SCV values. It is interesting to note, however, that for smaller buffer sizes in relation to the critical buffer size and increasing mean batch sizes, the mean queue length steadily improves with increasing values of the corresponding SCVs. This 'buffer size anomaly' can be attributed to the fact that, for a given arrival rate, the mean batch size of arriving bulks increases whilst the interarrival time between batches increases as the interarrival time SCV increases, resulting in a greater proportion of arrivals being blocked (lost) and, thus, a lower mean effective arrival rate; this influence has much greater impact on smaller buffer sizes.

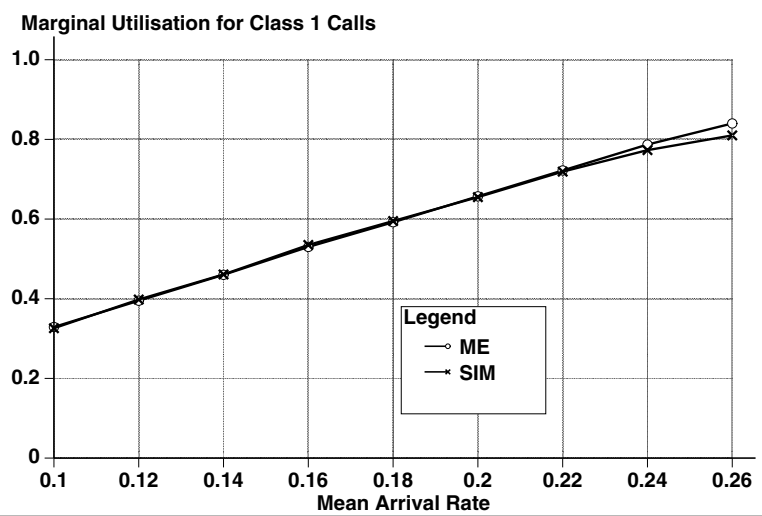

Fig. 2. Marginal Utilisations for Class 1 Calls

\section{Conclusions}

A novel analytic framework is presented for the performance modelling and evaluation of a wireless GSM/GPRS cell with both voice and multiple data services under a CPS, a pessimistic limiting case of the PSS. The proposed model is comprised from two independent queueing systems, namely an $\mathrm{M} / \mathrm{M} / \mathrm{c} / \mathrm{c}$ loss system with Poissonian GSM traffic and a GE/GE/1/N $/$ FCFS $\rightarrow$ GE $/ \mathrm{GE} / 1 / \mathrm{N}_{2} / \mathrm{PS}$ 


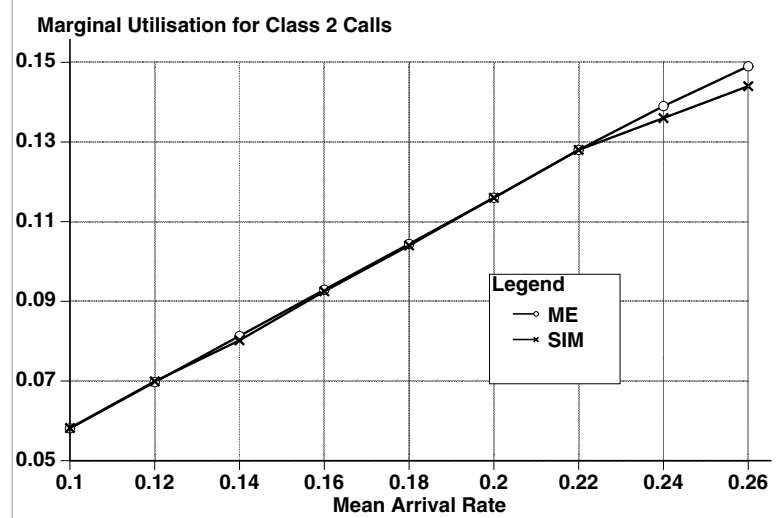

Fig. 3. Marginal Utilisations for Class 2 Calls

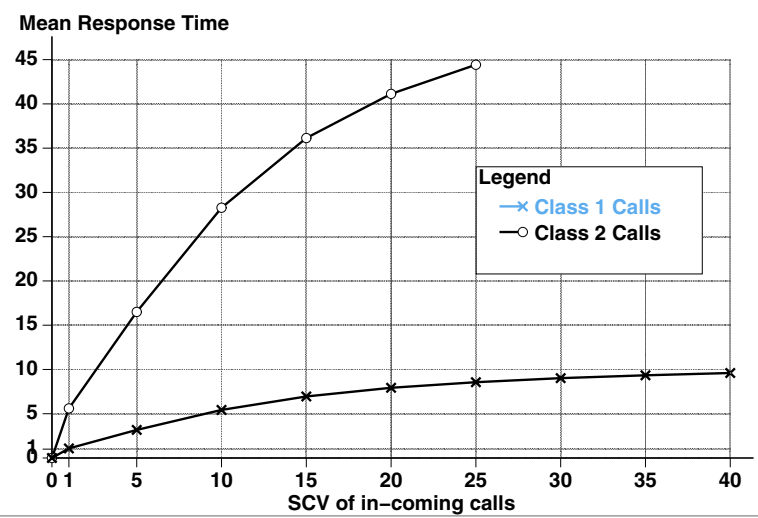

Fig. 4. Effect of varying degrees of SCV on Mean Response Time

system of access and transfer queues in tandem having a Compound Poisson external GPRS traffic with geometrically distributed batches.

The paper focuses on the analysis of the GE-type tandem system, which is valid for both uplink and downlink connections and provides voice and multiple class data services with different arrival rates and interarrival-time SCVs, file (burst) sizes and different PS discrimination service levels allowing a weighted capacity sharing. A product form approximation for the two queues in tandem is characterised, based on the principle of ME, leading into the decomposition of the system and the separate ME analysis of each building block queue under FCFS and PS rules, respectively, subject to GE-type queueing theoretic constraints per class. Subsequently, closed form expressions for state and blocking probabilities are established. Typical numerical examples are included to inves- 


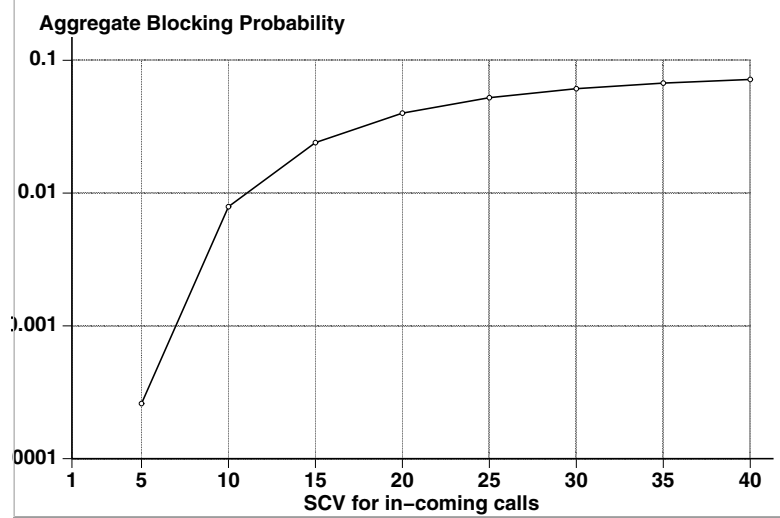

Fig. 5. Effect of varying degrees of SCV on Aggregate Blocking Probability

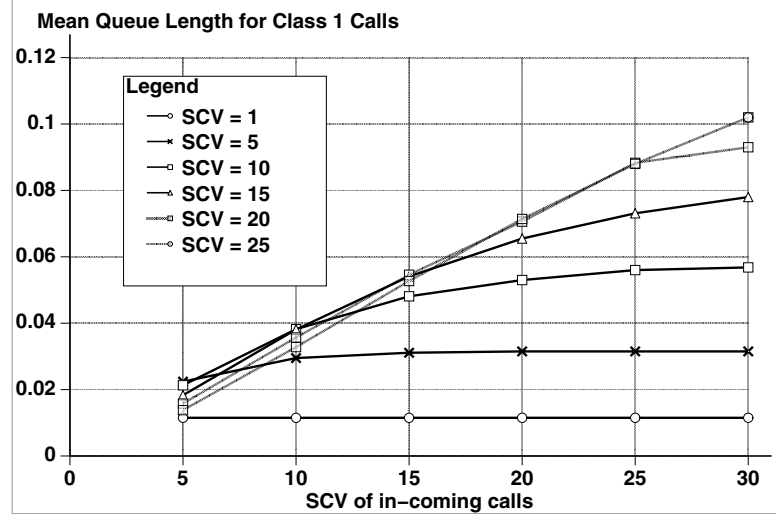

Fig. 6. Effect of varying degrees of SCV on MQLs of Class 1 at different buffer sizes

tigate the relative accuracy of the ME solution against simulation and to assess the effect of external GE-type bursty traffic upon the performance of the cell.

The paper has several extension possibilities. Firstly, the exponential assumption on the GSM call duration and interarrival time can be represented by a GE distribution resulting into a $\mathrm{GE} / \mathrm{GE} / \mathrm{c} / \mathrm{c}$ loss system. Secondly, the model can be generalised to capture the dynamics of data partition capacity under PSS. In this case, the blocked data calls at the transfer queue will be diverted towards the loss system which will be able to accommodate $\mathrm{R}+1$ classes (voice and data calls) under a preemptive resume (PR) priority rule (with voice having the highest priority). Finally, the ME methodology can be extended to model a network of multiple wireless cells using a QNM decomposition based on the principle of entropy maximisation. 


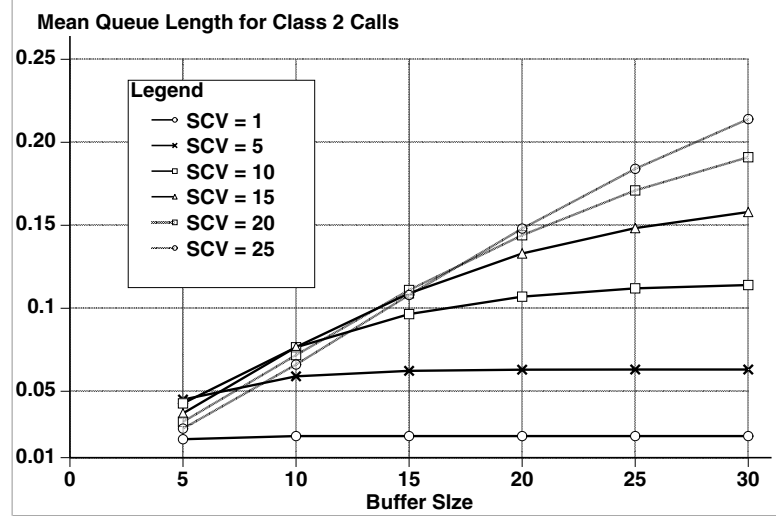

Fig. 7. Effect of varying degrees of SCV on MQLs of Class 2 at different buffer sizes

\section{References}

1. K. Begain, G. Bolch, M. Telek, Scalable Schemes for Call Admission and Handover Handling in Cellular Networks with Multiple Services. Journal on Wireless Personal Communications, Volume 15, No. 2, Kluwer Academic Publishers, 2000, pp. 125-144.

2. K. Begain, M.Ermel, T. Mueller, J. Schueller, M. Schweigel, Analytical Call Level Model of GSM/GPRS Network, in SPECTS'00, SCS Symposium on Performance Evaluation of Computer and Telecommunication Systems, Vancouver, BC, Canada, July 16-20, 2000.

3. R. Litjens, R. Boucherie, Radio Resource Sharing in GSM/GPRS Network. em ITC Specialist Seminar on Mobile Systems and Mobility, Lillehammer, Norway, March 22 - 24, 2000. pp. 261-274.

4. C.H.Foh, B.Meini, B. Wydrowski and M.Zuerman, Modeling and Performance Evaluation of GPRS, Proc. of IEEE VTC, 2001, Rhodes, Greece, pp. 2108-2112, May 2001.

5. D.D. Kouvatsos, P.H. Georgatsos and N.M. Tabet-Aouel, A Universal Maximum Entropy Algorithm for General Multiple Class Open Networks with Mixed Service Disciplines, Modelling Techniques and Tools for Computer Performance Evaluation, eds. R. Puigjaner and D. Potier, Plenum, pp 397-419, 1989.

6. D.D. Kouvatsos, Entropy Maximisation and Queueing Network Models, Annals of Operation Research, Vol. 48, pp. 63-126, 1994.

7. D.D. Kouvatsos and I.U.Awan, Open Queueing Networks with RS-Blocking and Multiple Job Classes, Research Report RR-08-01, Performance Modelling and Engineering Research Group, Department of Computing, Bradford University, August, 2001.

8. M. Veran and Potier D. QNAP-2, A Portable Environment for Queueing Network Modelling Techniques and Tools for Performance Analysis, D. Potier (ed.), North Holland, pp. 25-63, 1985. 